\title{
Data Fusion Models in WSNs: Comparison and Analysis
}

\author{
Marwah M Almasri, and Khaled M Elleithy, Senior Member, IEEE
}

\begin{abstract}
In WSNs, hundreds of sensors collect data from the environment but these sensors have limited energy. Therefore, energy consumption is a very challenging issue in the design of WSNs. Sometimes, sensors fail as they got affected by the pressure or temperature. Such failure can lead to misleading measurements which in turn are waste of energy. As a result, data fusion is needed to overcome such confusion where it assures data's efficiency and eliminates data's redundancy. This paper provides an analysis of the state-of-the-art data fusion models along with their architectures. It also presents a comparison between these models to highlight the main objectives of each. In addition, it analyzes the advantages and the limitation of these models.
\end{abstract}

Index Terms - Wireless Sensor Networks (WSNs); Data Fusion; Data Fusion Models; JDL Model; OODA Model; Intelligence Cycle Model; Omnibus Model; Object-Oriented Model.

\section{INTRODUCTION}

$\mathrm{W}$ ireless Sensor Networks (WSNs) have become a popular topic among researchers as it incorporates new technologies and perspectives. Therefore, it is crucial to pay attention to this type of network for exploring solutions to many problems. A WSN is composed of a huge number of sensors that are capable of observing the environment. However, due to limited computational power and energy of these sensors, we need to find ways to save energy. Data fusion is a great way that saves energy as it eliminates redundant and inaccurate data which are collected by malicious or failed sensors. Data fusion can be defined as the combination process of sensed data, where the resulted data are more accurate than each one individually [1]. Data fusion has been applied in many applications such as robotics and military applications [2], Denial of Service (DoS) detection [3], and sensor nodes' locations [4].

Due to the highly beneficial use of data fusion in WSNs, this paper provides detailed information about various data fusion models. Our goal is to distinguish each model and provide a

Manuscript received February 6, 2014.

M. M. Almasri is a Ph.D. candidate in the Computer Science \& Engineering Department at the University of Bridgeport, Bridgeport, CT, 06604. Phone: (203) 576-4703; e-mail: maalmasr@my.bridgeport.edu.

K. M. Elleithy is the Associate Dean for Graduate Studies in the School of Engineering at the University of Bridgeport, Bridgeport, CT, 06604. Phone: (203) 576-4703; e-mail: elleithy@bridgeport.edu. comparison between them. This paper also presents the advantages and the disadvantages of each model to understand the different objectives for applying such models.

This paper is structured as follows: section II, presents various data fusion models and their architectures. Section III provides a comparison between these models in order to distinguish the objectives of each one and evaluates them based on their advantages and drawbacks. Finally, section IV, concludes the paper.

\section{DATA FUSION MODELS}

Various data fusion models are proposed especially for the purpose of highlighting the specification, proposal, and usage of data fusion in WSNs [5]. These models can be categorized into three main models; data-based model, activity-based model, and role-based model as shown in Fig. 1. This section presents some of these models along with their architectures and designs.

\section{A. The JDL Model}

The JDL model is a popular model in the data fusion field. This model was introduced by the U.S. Joint Directors of Laboratories (JDL) and the U.S. Department of Defense (DoD) [6]. However, it has been revised by other researchers such as Steinberg et al [7]. The JDL model is one of the databased models that mainly focus on the abstraction level of the manipulated data by a fusion system. The JDL model composed of five major processing levels, a database management system, and a data bus that connects all components together. The architecture of the JDL model is represented in Fig. 2.

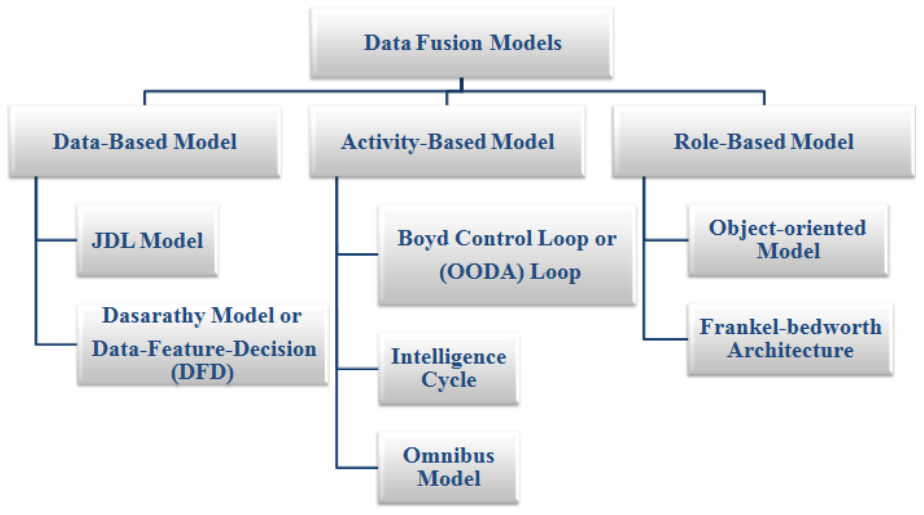

Fig. 1: Data Fusion Models. 


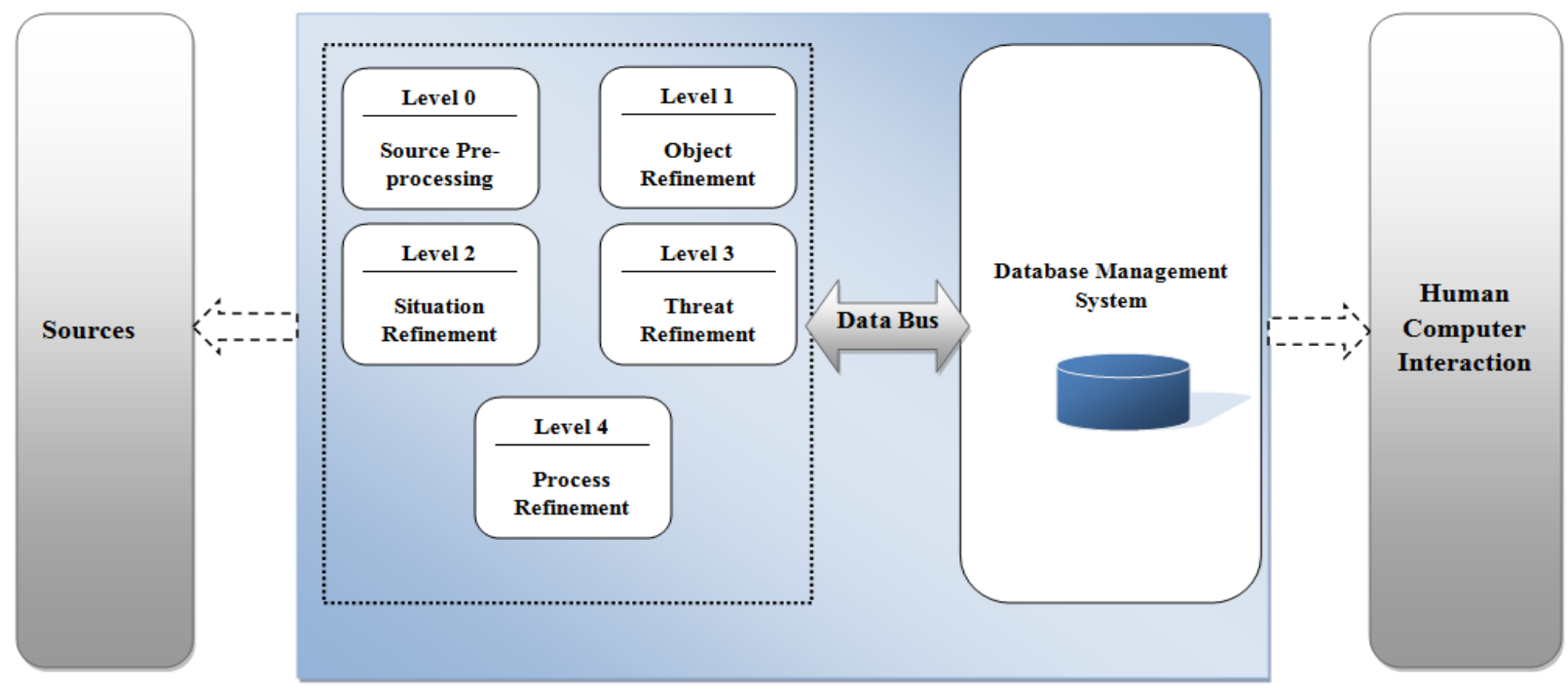

Fig. 2: The JDL Model

As shown in Fig. 2, sources are on the left side which demonstrate the inputs to the system. These sources can be local sensors, distributed sensors, or any other data either from a database or from human input [8].

In addition, on the right side, there is the Human Computer Interaction (HCI), which allows various kinds of human inputs such as data requests, reports, and commands. HCI provides multimedia methods for the purpose of interacting with human beings. The database management system is crucial as it is responsible for maintaining the fused data. It is also responsible for data retrieval, compression, and queries [8]. Level 0 of the JDL model called Source Preprocessing or Process Assignment. At this level, all data collected are assigned to appropriate processes which result in reducing the load in the data fusion system. Level 1 is called Object Refinement. Level 1 is responsible for transforming all kinds of sensors data into a consistent structure. It then assigns these data to objects in order to use the statistical estimation techniques in many applications. It also refines the predictions of an object's identity. Level 2 of the JDL model is called Situation Refinement. The Situation Refinement process describes the relationships between objects and events and emphasizes relational data. It interprets sensor data analogous to human's interpretation. It also examines level 1 results. Threat Refinement is referred to level 3 of the JDL model. It provides possible future threats and alternate hypotheses about enemies. Finally, level 4 which is called Process Refinement, is responsible for monitoring the performance of all data fusion processes in order to present a real time control. It also identifies what type of data is needed what are the source specific requirements for obtaining the set goals [8].

\section{B. Boyd Control Loop}

The Boyd Control Loop is one of the activity based models that are based on the executing of these activities in their correct sequence. It is also known as the Observe, Orient, Decide, Act (OODA) Loop. The Boyd Control Loop or the OODA Loop is a four stage cyclic model which describes the main activities in a fusion system [9] as shown in Fig. 3.

This model was proposed for decision-support in military system [9]. The first stage is the Observe stage which provides sensor preprocessing and data allocation. The second stage is the Orient stage which has all data alignment processes and situation predictions. The third stage is the Decide stage which is responsible for making decisions. The fourth and the last stage is the Act stage which includes the responsive system that executes the plan [10]. According to [11], the Observe stage of the OODA model corresponds to level 0 of the JDL model, where the Orient stage corresponds to level 1, 2, and 3 of the JDL model. In addition, the Decide stage corresponds to level 4 of JDL model. However, the Act stage is not included in the JDL model.

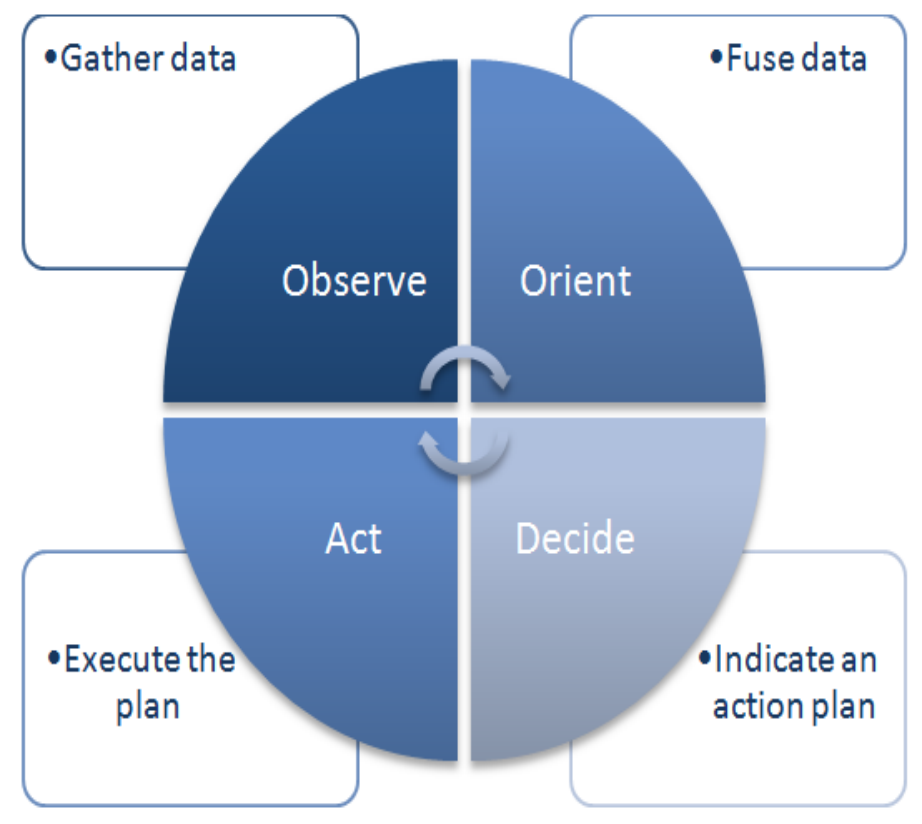

Fig. 3, The OODA model. 


\section{Intelligence Cycle}

The Intelligence Cycle is an activity based model that has a four stage cycle which describes the intelligence process for making decisions [12]. The four stages are as follows: Collection, Collation, Evaluation, and Dissemination as represented in Fig. 4. Collection stage collects raw intelligence data. Collation is responsible for collating reports to be ready for the next stage. At the Evaluation stage, the collated reports are fused and then analyzed, where at the Dissemination stage, users can use the results from previous stage in order to make decisions [13].

\section{Omnibus Model}

The Omnibus model is an activity based model. It was first proposed by [11]. The Omnibus model is shown in Fig. 5 which composed of four main stages. Sensing and Signal Processing stage is responsible for collecting and preprocessing data. At the Feature Extraction stage, patterns are extracted from the gathered data. After pattern extraction is complete, these patterns are further fused. At the Decision stage, decisions are made and threats are tracked, where the best plan is chosen and executed at the Act stage [14].

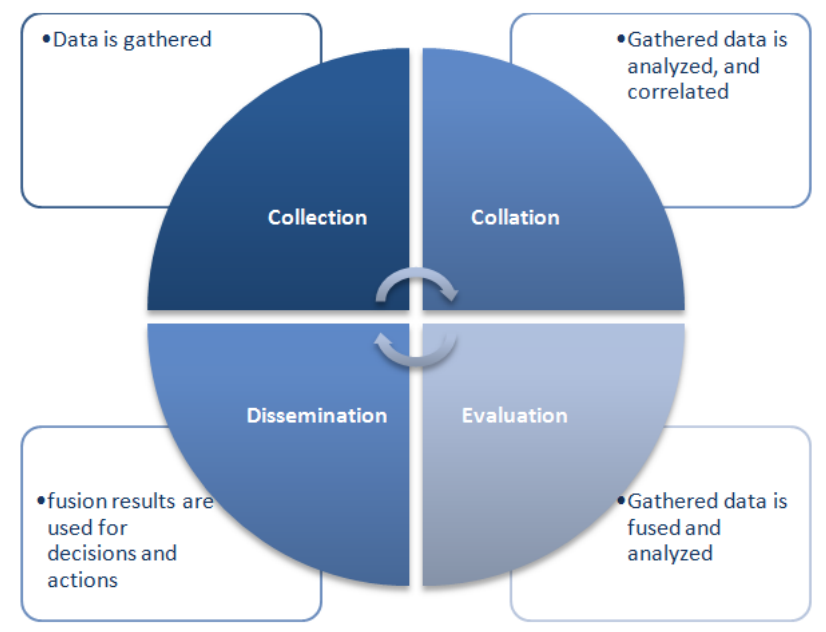

Fig. 4: The Intelligence Cycle Model.

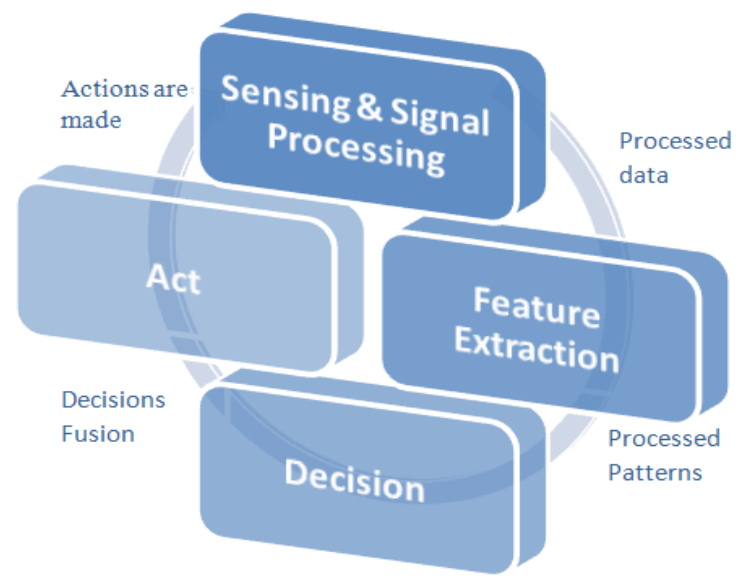

Fig. 5: The Omnibus Model.

\section{E. Object-Oriented Model}

The Object-Oriented model is a role based model that has a cyclic architecture based on roles. This model was proposed by Kokar et. al. [15]. Fig. 6 gives an overview of the ObjectOriented model. These roles as follows: the Actor has the role of interacting with the world, the Perceiver has the role of evaluating and analyzing the collected data, the Director has the role of creating plans based on the system set goals, and finally the Manger has the role of performing the set plans by the director [14].

\section{COMPARISON AND ANALYSIS OF DATA FUSION MODELS}

As shown in Table I, there are several factors to compare and contrast the data fusion models, where it relates different stages from each model to comprehend the objective of every process within the data fusion domain. These factors as follows:

- Data Gathering: this explains how the data will be collected and at which stage.

- Signal Processing: this means the preprocessing of data and data allocation before the fusion process begins.

- Object Assessment: after preprocessing the data, and patterns and features are extracted, these data are assigned to objects.

- Situation Assessment: it includes the situation predictions, the data fusion process, and the analysis of results.

- Threat Assessment: it indicates the possible threats.

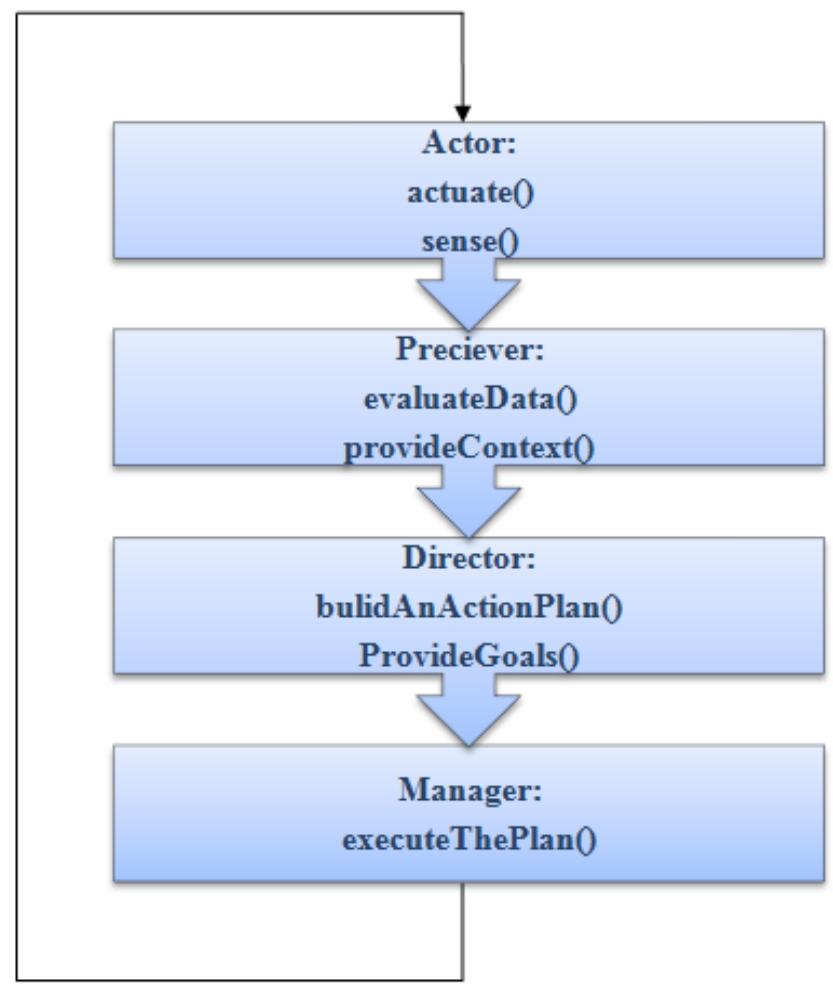

Fig. 6: The Object-Oriented Model. 
Table I, comparison between data fusion models
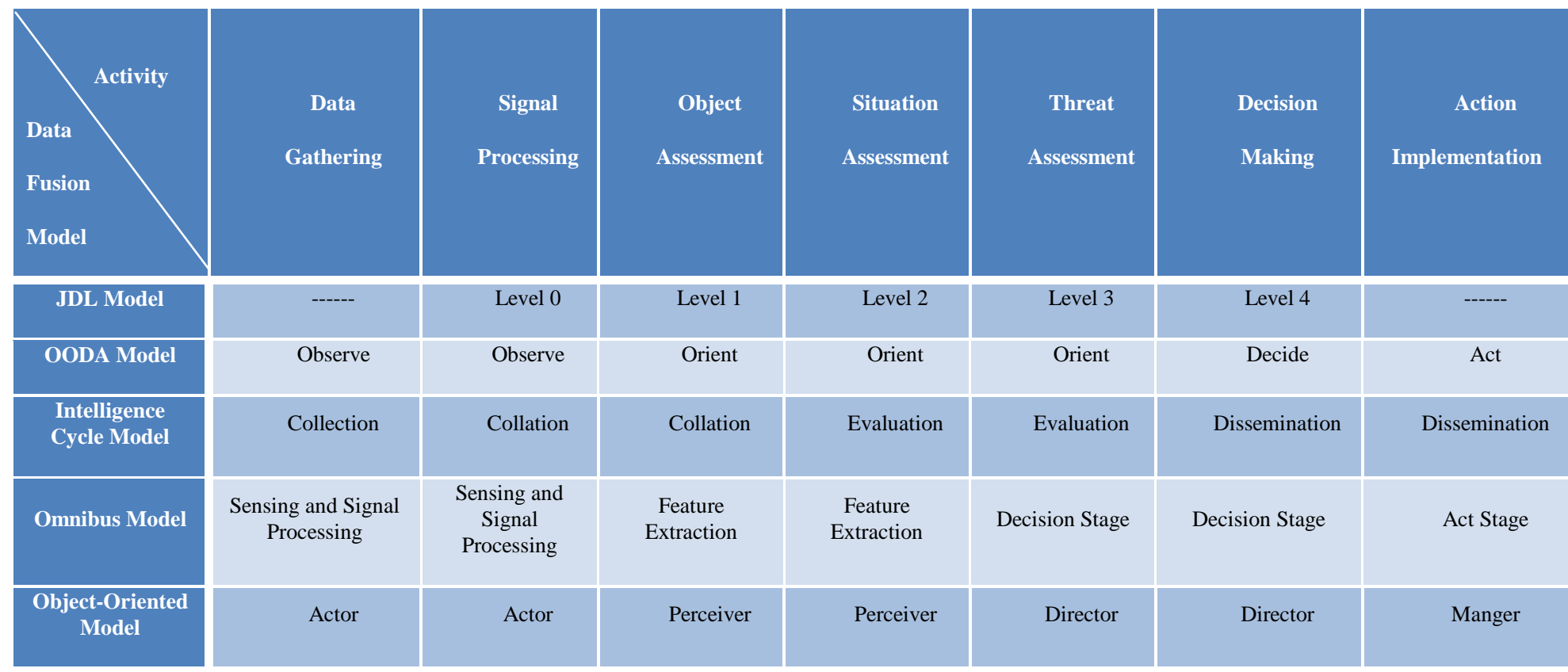

Collation

Eva

Actor

Actor

Feature
Extraction

\begin{tabular}{|l|l|l|}
\hline Evaluation & Evaluation \\
\hline
\end{tabular}

\begin{tabular}{c|c} 
Feature \\
Extraction
\end{tabular}

\begin{tabular}{|l|l|l|l|l|}
\hline Perceiver & Perceiver & Director & Director & Manger \\
\hline
\end{tabular}

- Decision Making: it states at which stage the decision making process is involved either by the users or by the system. It also provides the best plan to be implemented at the following stage.

- Action Implementation: this explains the actual plan execution.

As indicated in Table $\mathrm{I}$, the data gathering process is applicable at all models except at the JDL model. The JDL model starts with the Source Preprocessing stage (Level 0), which assumes that the data is already collected and now it is ready for the preprocessing phase. The data gathering or data/sensor collection corresponds to Observe stage of the OODA model, to Collection stage of the Intelligence Cycle Model, to Sensing stage of the Omnibus Model, and finally to Actor role of the Object-Oriented Model. For signal processing, all models will preprocess data at early stages. Most of the models gather data and process them at the same stage except for the JDL model and the Intelligence Cycle Model, where they do separate these processes at two different stages. Object Assessment is done at Level 1 of the JDL model, Orient stage of the OODA model, Collation stage of the Intelligence Cycle Model, Feature Extraction of the Omnibus model, and at the Perceiver role of the Object-Oriented Model. Situation Assessment process is done at most models as the Object Assessment is taking place such as the OODA Model, the Omnibus Model, and the Object-Oriented Model. In contrast, the Situation Assessment is applied at different stages from the Object Assessment in the JDL model (level 2), and the Intelligence Cycle Model (Evaluation stage).
In addition, Threat Assessment is implemented sometimes as an evaluation process after data is fused, or as a decision needs to be made. It is done at Level 3 of the JDL model, the Orient stage of the OODA model, at Evaluation of the Intelligence Cycle Model, at Decision Stage of the Omnibus model, and at the Director role of the Object-Oriented Model.

Decision Making and action implementation are done mostly at different stages where both take place at the end of each model. For the JDL model, the action implementation is not applicable. However, at the Intelligence Cycle Model, the action implementation and decision making process are done at the same stage which is the Dissemination stage.

As a result, we can see how different stages from different data fusion models are overlapped as the process or activity develops from sensing and collecting data to implementing actions and executing commands. This emphasizes the general and the specific objectives of all fusion models. Some models have more general goals such as the JDL model, where it focuses on the fusion process more than acquiring data and implementing commands. Others are more like in depth process such as the Omnibus model. It has all components needed for an effective data fusion model as it still has the cyclic loop like other models but it considers the importance of having a feedback explicitly in the system.

Furthermore, Table II, concludes and summarizes the advantages and the disadvantages of the data fusion models discussed in this paper. For using any data fusion model, it is important to keep in mind the purpose of using such a model in terms of requirements and future use. Some models are difficult to be reused at different applications such as the JDL Model and the Omnibus Model due to its sophisticated techniques used or due to its adjustments to specific 
Table II, summarizes the advantages and the limitations of the data fusion models.

\begin{tabular}{|c|c|c|}
\hline Data Fusin Model & Advantages & Limitations \\
\hline JDL Model & $\begin{array}{l}\text { Popular and general data fusion } \\
\text { model. }\end{array}$ & $\begin{array}{l}\text { Difficult to reuse the model after applying it at } \\
\text { specific application as it does not specify the } \\
\text { techniques used. }\end{array}$ \\
\hline OODA Model & $\begin{array}{l}\text { It has the ability to separate the } \\
\text { system tasks clearly and gives a } \\
\text { feedback. }\end{array}$ & $\begin{array}{l}\text { It does not show the effect of the Act stage at other } \\
\text { stages of the model. }\end{array}$ \\
\hline $\begin{array}{l}\text { Intelligence Cycle } \\
\text { Model }\end{array}$ & General data fusion model. & Does not separate the system tasks. \\
\hline Omnibus Model & $\begin{array}{l}\text { It explicitly describes the } \\
\text { processing levels in the cyclic } \\
\text { loop. }\end{array}$ & $\begin{array}{l}\text { If there is a specific requirement needed for an } \\
\text { application, this model is not the best data fusion } \\
\text { model as it combines several phases such as soft and } \\
\text { hard decisions which lead to a confusion. }\end{array}$ \\
\hline $\begin{array}{l}\text { Object-Oriented } \\
\text { Model }\end{array}$ & $\begin{array}{l}\text { Describes the various roles of the } \\
\text { system. }\end{array}$ & Does not separate the system tasks. \\
\hline
\end{tabular}

application requirements which makes it complex to reapply. Other data fusion models are more general such as the OODA Model and the Intelligence Cycle Model.

\section{CONCLUSION}

Data fusion has a vital role in WSNs as it saves the energy consumed within the network due to its ability of eliminating redundant data and thus increasing the accuracy and the efficiency of the sensed data. In this paper, we investigate different data fusion models and provide a comparison between them based on several factors. We also highlight the benefits and the drawbacks of each data fusion model presented in this paper in order to understand the objective of applying each model.

\section{REFERENCES}

[1] W. Elmenreich, "Sensor Fusion in Time-Triggered Systems", PhD thesis, Technische Universit"at Wien, Institut f'ur Technische Informatik, Vienna, Austria, 2002

[2] R. Brooks and S. Iyengar, "Multi-Sensor Fusion: Fundamentals and Applications with Software", Prentice Hall PTR, Upper Saddle River, NJ, 1998

[3] C. Siaterlis and B. Maglaris, "Towards multisensor data fusion for DoS detection", In Proceedings of the 2004 ACM Symposium on Applied Computing. ACM Press, Nicosia, Cyprus, pp. 439-446, 2004.

[4] A. Savvides, C. Han, and M. Strivastav, "The n-hop multilateration primitive for node localization”, Mobile Netw. Appl. 8, pp. 443-451, August 2003.

[5] E. Nakamura, A. Loureiro, and A. Frery, "Information fusion for wireless sensor networks: methods, models, and classifications", ACM Comput. Surv., vol. 39, no. 3, 2007. Article 9.

[6] O. Kessler et al., " Functional description of the data fusion process", Tech. rep., Naval Air Development Center, Warminster, PA, USA. January. Report prepared for the Office of Naval Technology. 1992

[7] A. N. Steinberg, C. L. Bowman, and F. E. White, "Revisions to the JDL data fusion model," in AeroSense'99, pp. 430-441, 1999.

[8] D. Hall, and J. Llinas, "An introduction to multi-sensor data fusion", Proc. IEEE, vol. 85, no. 1, pp. 6-23, January 1997.
[9] J. R. Boyd, "The essence of winning and losing," Unpublished lecture notes, 1996.

[10] T. Määttä, "Sensor fusion in smart camera networks for Ambient IntelligenceReport on PhD Thesis and Defense," Journal of Ambient Intelligence and Smart Environments, vol. 5, pp. 415-417, 2013.

[11] M. Bedworth and J. O'Brien, "The Omnibus model: a new model of data fusion?", IEEE Aerospace and Electronic Systems Magazine, April 2000, pp. 30-36.

[12] A. Shulsky and G. Schmitt, " Silent Warfare: Understanding the World of Intelligence”, 3 ed. Brasseys, Inc., New York, NY. 2002.

[13] C. Frankel and M. Bedworth, "Control, estimation and abstraction in fusion architectures: Lessons from human information processing", in Proceedings of the $3^{\text {rd }}$ International Conference on Information Fusion (Fusion 2000). Vol. 1. IEEE, Paris, France, MoC5/3-MoC5/10, 2000.

[14] V. Borges and W. Jeberson, "Survey of Context Information Fusion for Sensor Networks based Ubiquitous Systems", in J. Sens. Actuator Netw., 2013.

[15] M. M. Kokar, M. D. Bedworth, and C. B. Frankel, "Reference Model for data fusion Systems", in AeroSense 2000, pp. 191-202, 2000.

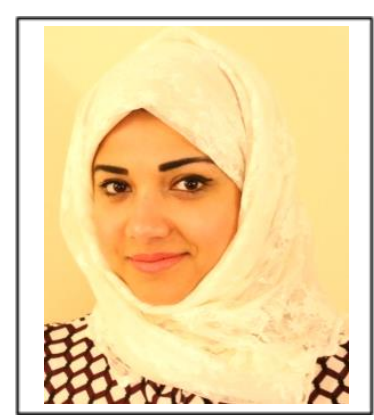

Mrs. Marwah M Almasri: is a Ph.D. candidate in the Computer Science \& Engineering Department at the University of Bridgeport. She received award from UPE for her academic accomplishments in the fields of the computing and information disciplines. She received her MBA in Management Information System (MIS) from the University of Scranton, PA, in 2011. She also received award from MIS department at the University of Scranton for her outstanding work.

She holds a bachelor degree in Computer Science \& Engineering from Taibah University in Medina, Saudi Arabia. Her research interests include congestion mechanisms, wireless sensor networks, computer networks, mobile computing, network security, and data fusion. 


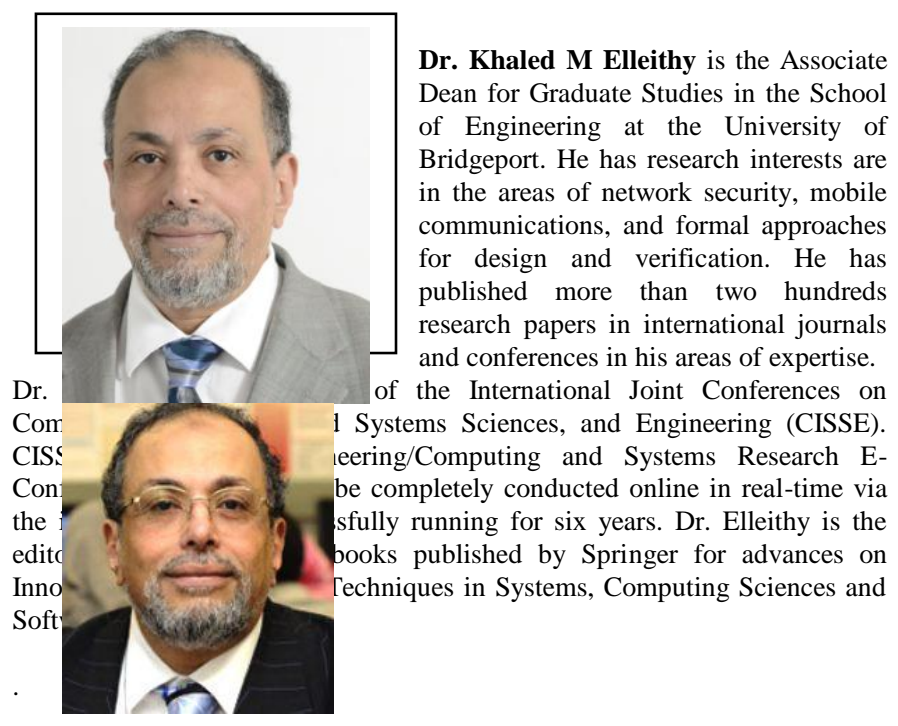

